\title{
Upregulation of long non coding RNA PCAT-1 contributes to cell proliferation, migration and apoptosis in hepatocellular carcinoma
}

\author{
JIFENG WEN ${ }^{1}$, JUN XU $^{1}$, QIFENG SUN ${ }^{1}$, CHENGLIANG XING $^{1}$ and WENZHE YIN ${ }^{2}$ \\ Departments of ${ }^{1}$ Gastroenterology and ${ }^{2}$ Orthopedic Surgery, The Second Affiliated Hospital, \\ Harbin Medical University, Harbin, Heilongjiang 150086, P.R. China
}

Received May 22, 2015; Accepted March 21, 2016

DOI: $10.3892 / \mathrm{mmr} .2016 .5075$

\begin{abstract}
Long non-coding RNAs (lncRNAs) exert regulatory functions on various biological processes in cancer cells, including proliferation, apoptosis and mobility. Prostate cancer-associated transcript 1 (PCAT-1) is a novel lncRNA that promotes cell proliferation in prostate cancer, however, the effect of PCAT-1 in hepatocellular carcinoma (HCC) remains to be elucidated. The present study hypothesized that PCAT-1 also exerts an important effect in HCC. The current study investigated PCAT-1 expression levels in HCC tissue samples and HepG2 and Bel-7402 cell lines using the reverse transcription-quantitative polymerase chain reaction. The results demonstrated that PCAT-1 was upregulated in HCC tissue samples and cell lines compared with adjacent non-cancerous tissues and the L02 normal liver epithelial cell line. PCAT-1 suppression using PCAT-1 small hairpin RNA in HepG2 and Bel-7402 cells inhibited cell proliferation and migration, and induced apoptosis. Overexpression of PCAT-1 induced synthetic plasmid vectors was demonstrated to increase cell proliferation and migration, and inhibit apoptosis. Results from the present study suggest that PCAT-1 exerts an oncogenic effect in HCC and silencing PCAT-1 may be a potential novel therapeutic strategy for HCC.
\end{abstract}

\section{Introduction}

Hepatocellular carcinoma (HCC) is the predominant histologic subtype of primary liver cancer, accounting for 70-85\% of cases in the majority of countries, and is a leading cause of cancer-associated mortality each year worldwide $(1,2)$. Although a number of advances in surgical techniques and medical care have been achieved over the last two decades, long-term survival of HCC remains low due to the high rate of

Correspondence to: Dr Wenzhe Yin, Department of Orthopedic Surgery, The Second Affiliated Hospital, Harbin Medical University, 246 Xuefu Road, Harbin, Heilongjiang 150086, P.R. China

E-mail: yinwenzhe007@163.com

Key words: long non-coding RNAs, hepatocellular carcinoma, prostate cancer-associated transcript 1 , oncogenic recurrence and metastasis $(3,4)$. Furthermore, the majority of HCC patients present with advanced metastatic disease at the initial diagnosis. HCC develops via a complex process associated with multi-step genetic and epigenetic changes $(5,6)$. Thus, it is key to develop novel approaches to aid early diagnosis, prognosis determination, and therapeutic strategies to treat HCC.

Non-coding RNAs (ncRNAs) are sub-divided into two major classes according to size, small ncRNAs are $<200 \mathrm{nt}$ in length and long ncRNAs are $>200 \mathrm{nt}$ in length (7). In recent years, microRNAs have been identified as oncogenes or tumor suppressor genes due to the effects exerted on cancer cells via post-transcriptional regulation of protein expression (8-10). By contrast, IncRNAs were previously hypothesized to be transcriptional noise. However, lncRNAs are key in important functions at numerous levels, including $\mathrm{X}$ chromosome inactivation, chromatin remodeling, and transcriptional repression (11-13).

With the development of deep sequencing technologies, IncRNAs have increasingly been associated with various human diseases, particularly in types of cancer (14-16). LncRNAs exert important regulatory effects in the programming of cellular functions and may be potential oncogenes or tumor suppressor RNAs (17). Prostate cancer-associated ncRNA transcripts 1 (PCAT-1) is a long non-coding RNA that was originally identified as a biomarker for prostate cancer (18). High expression levels of PCAT-1 were also observed to be involved in the progression of colorectal cancer (19), and upregulation of PCAT-1 has also been reported to be associated with advanced clinical stage and poor prognosis in esophageal squamous carcinoma (20). However, the role of PCAT-1 in HCC remains to be elucidated.

In the present study, PCAT-1 was demonstrated to be crucial in the regulation of proliferation, migration and apoptosis of HCC cells. To the best of our knowledge, this is the first study that directly illustrates the role of PCAT-1 in HCC. Thus, PCAT-1 may serve as a potential prognostic biomarker and therapeutic target in HCC.

\section{Materials and methods}

Patient samples. HCC tissue samples and adjacent morphologically normal tissue samples were collected from 82 patients enrolled in the present study who underwent surgery between 
August 2009 and May 2013 at the Second Affiliated Hospital, Harbin Medical University (Harbin, China). None of the patients had received percutaneous ablation, chemoembolization or radiotherapy prior to the surgery. The diagnosis was based on detailed examination of sections stained with hematoxylin and eosin and was confirmed in all cases by the Department of Pathology of The Second Affiliated Hospital. The present study was approved by the Institutional Review Board of The Second Affiliated Hospital and written informed consent was obtained from all patients. Specimens were obtained immediately following surgical resection and stored at $-80^{\circ} \mathrm{C}$ for further analysis.

Cell culture. The HepG2 and Bel-7402 hepatocellular carcinoma cell line and the L02 normal liver epithelial cell line were purchased from the Institute of Biochemistry and Cell Biology, Chinese Academy of Science (Shanghai, China). All cell lines was cultured in Invitrogen Dulbecco's modified Eagle's medium (DMEM; Thermo Fisher Scientific, Inc., Waltham, MA, USA) supplemented with $10 \%$ fetal bovine serum (FBS; Invitrogen; Thermo Fisher Scientific, Inc.) at $37^{\circ} \mathrm{C}$ in a $5 \% \mathrm{CO}_{2}$ atmosphere.

Cell transfection. The cells were cultured in a 6-well or 24-well plates for $24 \mathrm{~h}$ and then transfected with overexpression plasmid vectors or small hairpin (sh)RNAs. All transfections were performed using Lipofectamine 2000 reagent (Invitrogen; Thermo Fisher Scientific, Inc.) according to the manufacturer's protocol. PCAT-1 shRNA and negative control shRNA were obtained from Shanghai GenePharma Co., Ltd. (Shanghai, China) with the sequence 5'-GAGAAAGCAUCU GUACCCUUACAAU-3'. The overexpression plasmid vector for PCAT-1, PCAT-1-pcDNA3.1 + vector, was supplied and synthesized by Invitrogen (Thermo Fisher Scientific, Inc.). The pcDNA3.1 + empty vector was used as a negative control.

Reverse transcription-quantitative polymerase chain reaction $(R T-q P C R)$. Total RNA was extracted from the tissue samples or the transfected cells using TRIzol (Invitrogen; Thermo Fisher Scientific, Inc.) according to the manufacturer's protocol. RNA was reverse transcribed using SuperScript First Strand cDNA system (Invitrogen; Thermo Fisher Scientific, Inc.) according to the manufacturer's protocols. The temperature protocol was as follows: $65^{\circ} \mathrm{C}$ for $5 \mathrm{~min}, 4^{\circ} \mathrm{C}$ for $1 \mathrm{~min}, 50^{\circ} \mathrm{C}$ for $50 \mathrm{~min}$ and finally $85^{\circ} \mathrm{C}$ for $5 \mathrm{~min}$. Thirty-six cycles of qPCR were performed at $98^{\circ} \mathrm{C}$ for $2 \mathrm{~min}, 98^{\circ} \mathrm{C}$ for $10 \mathrm{sec}, 60^{\circ} \mathrm{C}$ for $30 \mathrm{sec}$, and $72^{\circ} \mathrm{C}$ for $30 \mathrm{sec}$; the final stage was performed at $72^{\circ} \mathrm{C}$ for $5 \mathrm{~min}$. The primer sequences were as follows: Sense, $5^{\prime}$-AAT GGCATGAACCTGGGAGGCG-3' and antisense, 5'-GGCTTT GGGAAGTGCTTTGGAG-3' for PCAT-1; and sense, 5'-ACA TCAAGAAGGTGGTGAAGCAGG-3' and antisense, 5'-CGT CAAAGGTGGAGGAGTGGGT-3' for GAPDH (Invitrogen; Thermo Fisher Scientific, Inc.. qPCR was performed using the SYBR ${ }^{\circledR}$ Premix Ex Taq ${ }^{T M}$ II PCR kit (Takara Bio, Inc., Otsu, Japan) according to the manufacturer's protocols on the ABI Prism ${ }^{\circledR} 7000$ Real-Time PCR system (Applied Biosystems; Thermo Fisher Scientific, Inc.). Data was collected and analyzed by SDS 2.3 software (Applied Biosystems; Thermo Fisher Scientific, Inc.). The expression level of PCAT-1 was normalized using the $\mathrm{Cq}$ of the housekeeping gene GAPDH. The relative quantitative value was expressed using the $2^{-\Delta \Delta \mathrm{Cq}}$ method (21). Each experiment was performed in triplicate and repeated three times.

Cell Counting Kit-8 (CCK-8) assay. Cell proliferation was detected using the CCK-8 assay (Dojindo Molecular Technologies, Kumamoto, Japan), according to the manufacturer's protocols. Cells were seeded in 96-well plates and cultured in normal medium (DMEM + FBS) for $24 \mathrm{~h}$ prior to transfection with PCAT-1 shRNA or negative control shRNA and PCAT-1-pcDNA3.1 + vector or pcDNA3.1 + empty vector. At $0,24,48$, and $72 \mathrm{~h}$ after transfection, the absorbance of each well at a wavelength of $450 \mathrm{~nm}$ was measured by a microplate reader (Bio-Rad Laboratories, Inc., Hercules, CA, USA). All experiments were performed three times.

Cell migration assay. Following washing with phosphate-buffered saline (Sigma-Aldrich, St. Louis, MO, USA), HepG2 or Bel-7402 cells were detached with trypsin (Sigma-Aldrich), and resuspended in serum-free medium (DMEM). The cell suspensions $\left(200 \mu \mathrm{l} ; 4 \times 10^{5}\right.$ cells $\left./ \mathrm{ml}\right)$ was added to the upper chamber with a non-coated membrane (EMD Millipore, Billerica, MA, USA) for the Transwell migration assays (Costar; Corning Life Sciences, Cambridge, MA, USA). Culture medium containing 10\% FBS was added to the bottom wells of the chambers. Following incubation for $24 \mathrm{~h}$, the cells were removed using cotton swabs, fixed with $100 \%$ methanol (Sigma-Aldrich) and stained with $0.2 \%$ Crystal Violet (Sigma-Aldrich). Images were acquired using a Leica DMI 6000B microscope (magnification, x100; Leica Microsystems Inc., Richmond Hill, ON, Canada).. The assays were repeated at least three times.

Caspase-3 ELISA assay. HepG2 and Bel-7402 cells were transfected with PCAT-1 shRNA or negative control shRNA, and PCAT-1-pcDNA3.1 + vector or pcDNA3.1 + empty vector. After $48 \mathrm{~h}$ transfection, apoptosis was determined by calculating the activity of caspase-3 using the Caspase- 3 ELISA activity assay kit (Invitrogen; Thermo Fisher Scientific, Inc.) according to the manufacturer's protocols. Optical density (OD) values were measured by using a microplate reader (Model 550; Bio-Rad Laboratories, Inc.). Data were presented as the ratios between the OD values of PCAT-1 shRNA-transfected cells or those of PCAT-1-pcDNA3.1 + vector-transfected cells and their respective controls. All experiments were performed in triplicate.

Statistical analysis. Data are presented as the mean \pm standard deviation. The difference in expression levels of PCAT-1 between HCC and matched healthy samples were analyzed using paired samples t-test. The differences between PCAT-1 shRNA or negative control shRNA-transfected cells and PCAT-1-pcDNA3.1 + vector or pcDNA3.1 + empty vector-transfected cells in the CCK- 8 assay was analyzed using analysis of variance. The independent samples t-test was used to analyze other data. Statistical analyses were performed using SPSS 17.0 (SPSS, Inc., Chicago, IL, USA). $\mathrm{P}<0.05$ was considered to indicate a statistically significant difference. 


\section{Results}

PCAT-1 expression levels were increased in hepatocellular carcinoma tissues and cell lines compared with controls. The relative expression level of PCAT-1 was determined using RT-qPCR in a total of 82 patient HCC samples. As presented in Fig. 1A, PCAT-1 was significantly upregulated in HCC tissue compared with matched normal tissue $(\mathrm{P}<0.05)$. The expression of PCAT-1 was also significantly increased in HepG2 and Bel-7402 cells compared with the L02 normal liver epithelial cell line (Fig. 1B; P<0.05). These results indicated that PCAT-1 may exert an oncogenic effect in HCC. Each experiment was performed in triplicate and repeated three independent times.

Silencing and overexpression of PCAT-1 was effectively induced by use of shRNA and overexpression vectors in HCC cell lines. HepG2 and Bel-7402 cells were cultured and transfected with PCAT-1 shRNA or negative control shRNA, and PCAT-1-pcDNA3.1 + vector or pcDNA3.1 + empty vector. At $48 \mathrm{~h}$ after transfection, the relative expression level of PCAT-1 was analyzed by qPCR. The results demonstrated that the relative expression level of PCAT-1 in HepG2 and Bel-7402 cells was significantly decreased by the PCAT-1 shRNA (Fig. 2A; P<0.05), while PCAT-1 was increased by the PCAT-1-pcDNA3.1+vector (Fig. 2B; P<0.05).

PCAT-1 increases proliferation in HCC cells. The present study also aimed to determine whether PCAT-1 promoted cell proliferation in HCC. HepG2 and Bel-7402 cells were transfected with PCAT-1 shRNA or negative control shRNA, and PCAT-1-pcDNA3.1 + vector or pcDNA3.1 + empty vector. The cell proliferation of the HCC cells were determined by CCK-8 assay. Transfection of PCAT-1 shRNA resulted in cell growth arrest in the HCC cells (Fig. 3A and $\mathrm{B} ; \mathrm{P}<0.05$ ), while transfection of PCAT-1-pcDNA3.1 + vector resulted in promoting cell proliferation (Fig. $3 \mathrm{C}$ and $\mathrm{D} ; \mathrm{P}<0.05$ ). These results demonstrated that PCAT-1 increases cell proliferation in HCC.

PCAT-1 exerts a positive effect on migration in HCC cells. To investigate the potential role of PCAT-1 in HCC metastasis, the present study detected whether PCAT-1 regulated the capacity of HCC cells to migrate. The cell migration assay demonstrated that cell migration was significantly inhibited in the groups transfected with PCAT-1 shRNA compared with those in the negative control shRNA group (Fig. 4A; $\mathrm{P}<0.05$ ). As presented in Fig. 4B, overexpression of PCAT-1 significantly promotes HCC cell migration $(\mathrm{P}<0.05)$. These data indicated that PCAT-1 has a positive effect on HCC cell migration.

Upregulation of PCAT-1 inhibited apoptosis in HCC cells. The current study also aimed to determine whether PCAT-1 inhibited cell apoptosis in HCC. HepG2 and Bel-7402 HCC cells were transfected with PCAT-1 shRNA or negative control shRNA, and PCAT-1-pcDNA3.1 + vector or pcDNA3.1 + empty vector. After transfection for $48 \mathrm{~h}$, the relative activity of caspase- 3 was determined by ELISA assay. As presented in Fig. 5, the relative activity of caspase- 3 was significantly increased by the PCAT-1 shRNA $(\mathrm{P}<0.05)$, while the relative activity of caspase-3 was
A

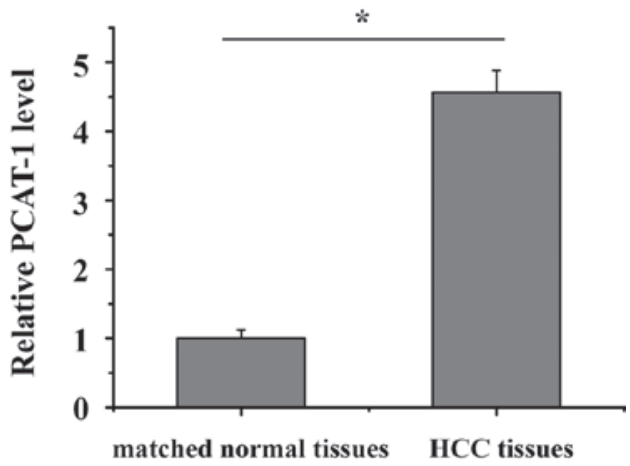

B

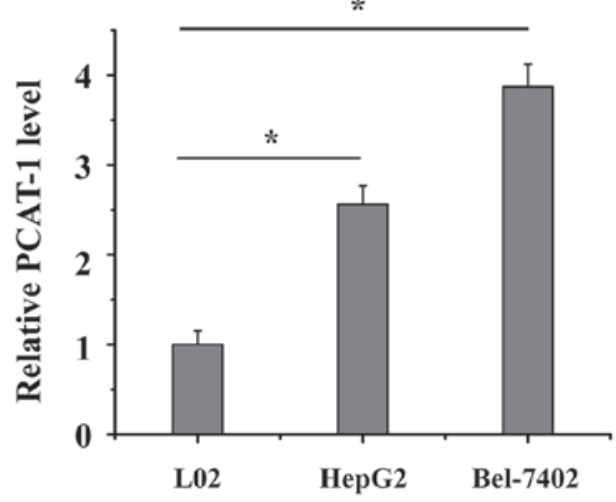

Figure 1. Expression levels of PCAT-1 in HCC tissue samples and cell lines. (A) Expression levels of PCAT-1 in 82 pairs of HCC tissue and matched normal tissue samples were measured by reverse transcription-quantitative polymerase chain reaction. GAPDH served as an internal control. (B) Expression levels of PCAT-1 in the L02 normal liver epithelial cell line and the HepG2 and Bel-7401 cell lines. Data were expressed as the mean \pm standard deviation $(n=3)$. Each experiment was performed in triplicate, three independent times. ${ }^{*} \mathrm{P}<0.05$. HCC, hepatocellular carcinoma; PCAT-1, prostate cancer-associated transcript 1.

significantly decreased by the PCAT-1-pcDNA3.1 + vector $(\mathrm{P}<0.05)$. These results suggest the upregulation of PCAT-1 inhibited HCC apoptosis and suggests that PCAT-1 exerts an oncogenic effect in hepatocellular carcinoma.

\section{Discussion}

LncRNAs represent an emerging group in hepatocellular carcinoma, they may exert effects on tumor proliferation and migration, and apoptosis (22-24). PCAT-1, a newly-identified lncRNA, is located in the chromosome $8 \mathrm{q} 24$ gene desert and contributes to cell proliferation in prostate cancer (18). Previous research has demonstrated that PCAT-1 may suppress the breast cancer 2, early onset tumor suppressor gene and produce a functional deficiency in homologous recombination in sporadic cancers (25). PCAT-1 promotes cell proliferation in prostate cancer via stabilization of the Myc proto-oncogene protein (26). A recent study demonstrated that silencing PCAT-1 may induce cell growth arrest and apoptosis in human bladder cancer (27).

The present study investigated the role of long non-coding RNA PCAT-1 in HCC. It was observed that the expression levels of PCAT-1 were upregulated in HCC tissue samples compared with matched normal tissue. Furthermore, PCAT-1 expression levels were also increased in the HepG2 and Bel-7402 HCC cell lines compared with the L02 normal liver 

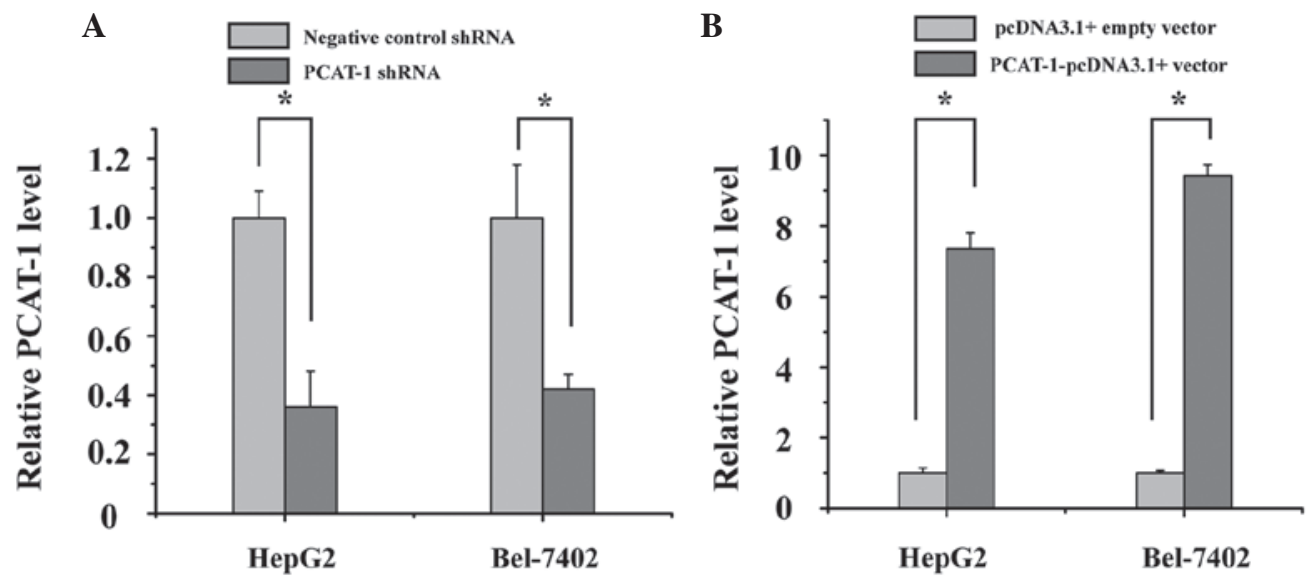

Figure 2. Silencing and overexpression of PCAT-1 in HCC cell lines. (A) The relative expression level of PCAT-1 was evaluated using reverse transcription-quantitative polymerase chain reaction. The PCAT-1-specific shRNA significantly decreased the expression level of PCAT-1 in HepG2 and Bel-7402 cells. (B) The PCAT-1-pcDNA3.1 + vector significantly increased the expression level of PCAT-1 in HepG2 and Bel-7402 cells. Data are presented as the mean \pm standard deviation $(\mathrm{n}=3)$. The experiments were performed in duplicate and repeated three times. " $\mathrm{P}<0.05$. PCAT1, prostate cancer-asssociated transcript 1; HCC, hepatocellular carcinoma; shRNA, small hairpin RNA.
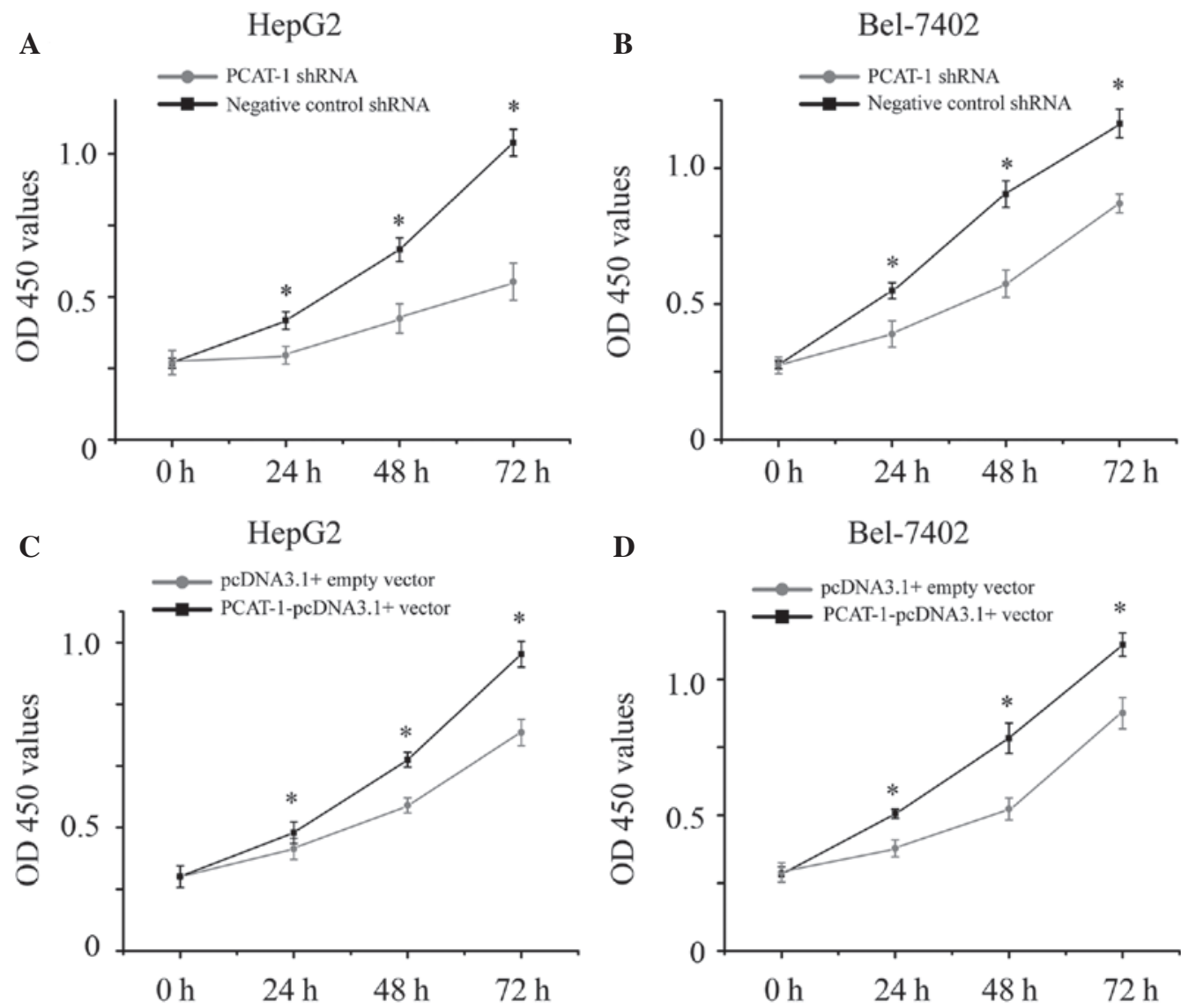

D

Bel-7402

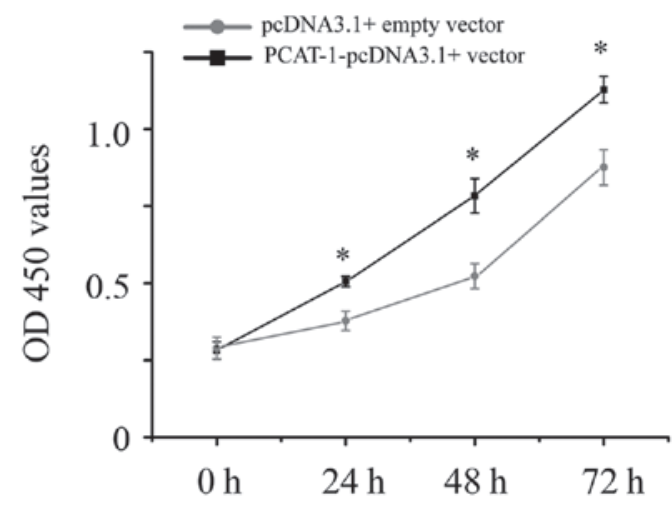

Figure 3. Effect of PCAT-1 on proliferation in HCC cells. The CCK-8 assay demonstrated significant cellular proliferation arrest in PCAT-1 shRNA-transfected (A) HepG2 and (B) Bel-7402 cells. The CCK-8 assay indicated significant acceleration of cell proliferation in PCAT-1-pcDNA3.1 + vector-transfected (C) HepG2 and (D) Bel-7402 cells. Data were expressed as the mean \pm standard deviation $(n=3)$. All experiments were performed three times, and a representative picture is presented. " $\mathrm{P}<0.05$. PCAT1, prostate cancer-asssociated transcript 1; HCC, hepatocellular carcinoma; shRNA, small hairpin RNA; CCK-8, Cell Counting Kit-8; OD, optical density.

epithelial cell line. These results suggest that PCAT-1 may exert an oncogenic effect in HCC development.

To further elucidate the biological functions of PCAT-1 in HCC, cell proliferation, migration and apoptosis were detected in cells following PCAT-1 silencing and overexpression in
HepG2 and Bel-7402 HCC cell lines. Arrest of cell proliferation, inhibition of migration and increased apoptosis were observed in PCAT-1 shRNA-transfected HCC cells. Furthermore, acceleration of cell proliferation, promotion of migration and decreased apoptosis were observed in 


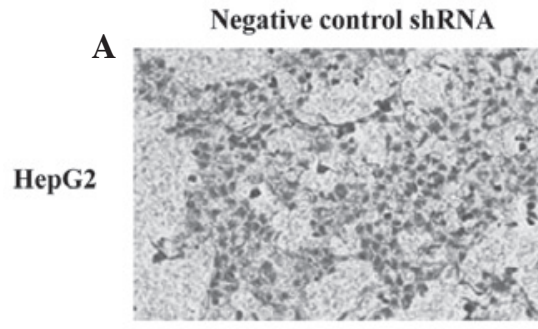

Negative control shRNA

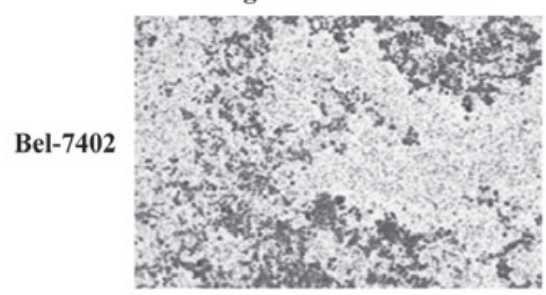

B
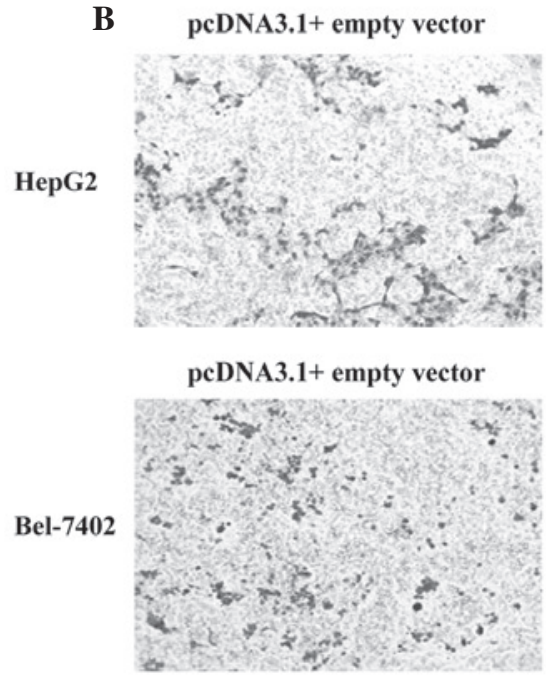

PCAT-1 ShRNA

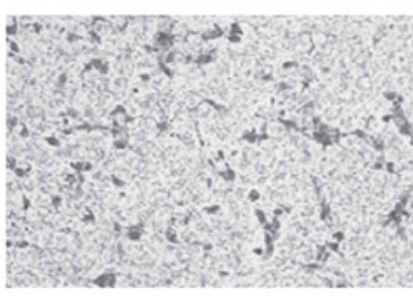

PCAT-1 ShRNA

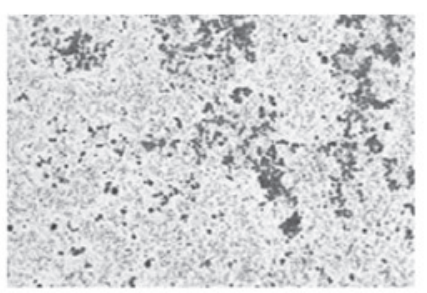

PCAT-1-pcDNA3.1+ vector

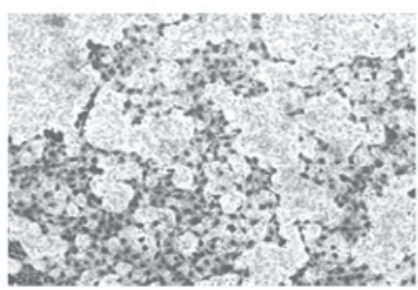

PCAT-1-pcDNA3.1+ vector

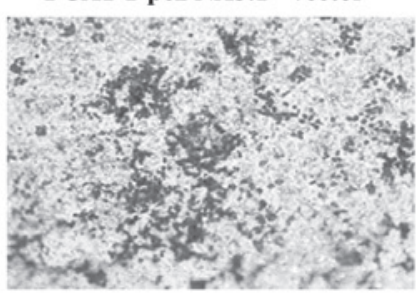

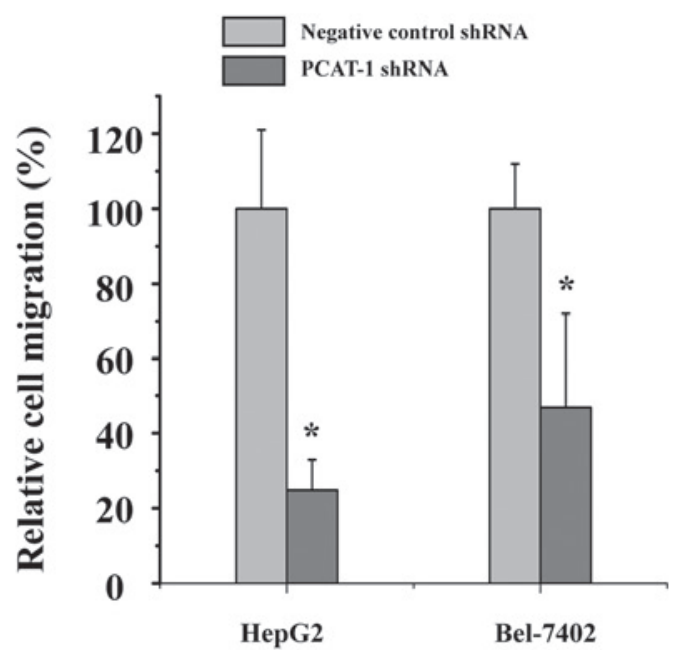

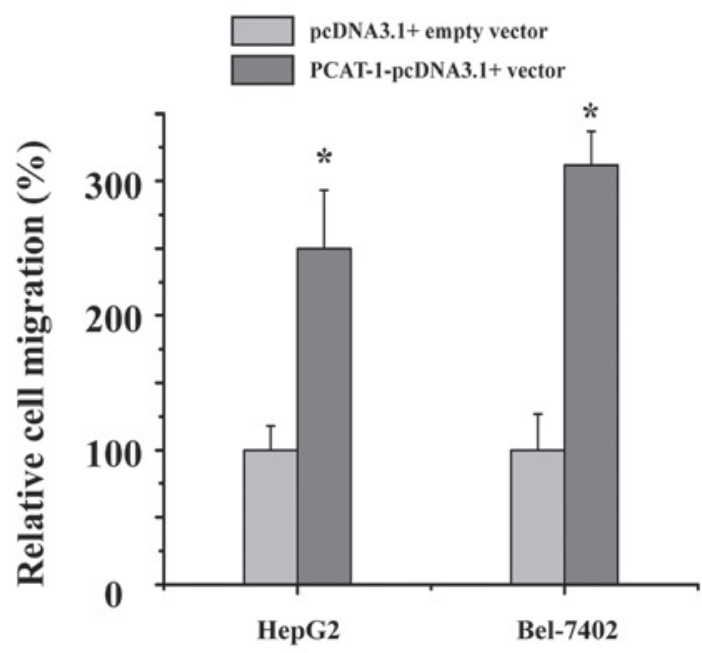

Figure 4. Effect of PCAT-1 on the migration in HCC cells. (A) The cell migration assay demonstrated significant inhibition of cell migration in PCAT-1 shRNA-transfected HepG2 and Bel-7402 cells. (B) The cell migration assay demonstrated significant promotion of cell migration in PCAT-1-pcDNA3.1 + vector-transfected HepG2 and Bel-7402 cells. Magnification, $\mathrm{x} 100$. Values were expressed as the mean \pm standard deviation ( $\mathrm{n}=3$ ). Each experiment was repeated three times with independent individuals. ${ }^{*} \mathrm{P}<0.05$. PCAT1, prostate cancer-asssociated transcript 1; HCC, hepatocellular carcinoma; shRNA, small hairpin RNA.

A

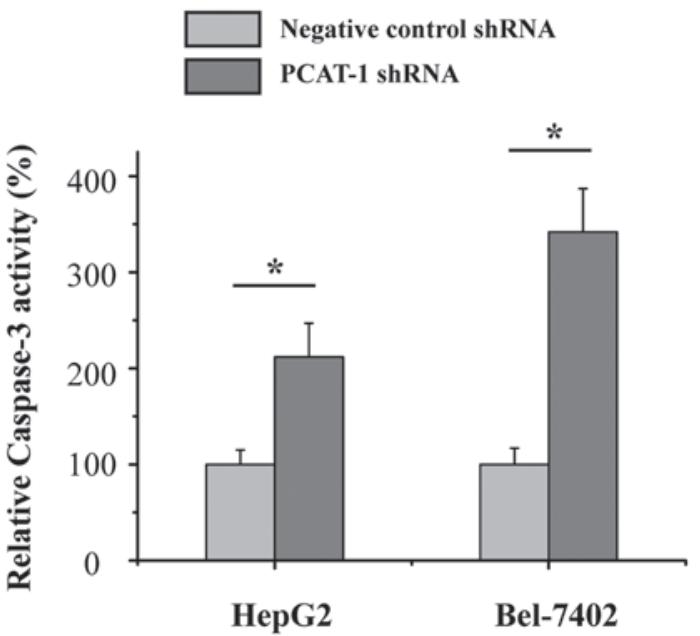

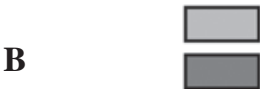

pcDNA3.1+ empty vector

PCAT-1-pcDNA3.1+ vector

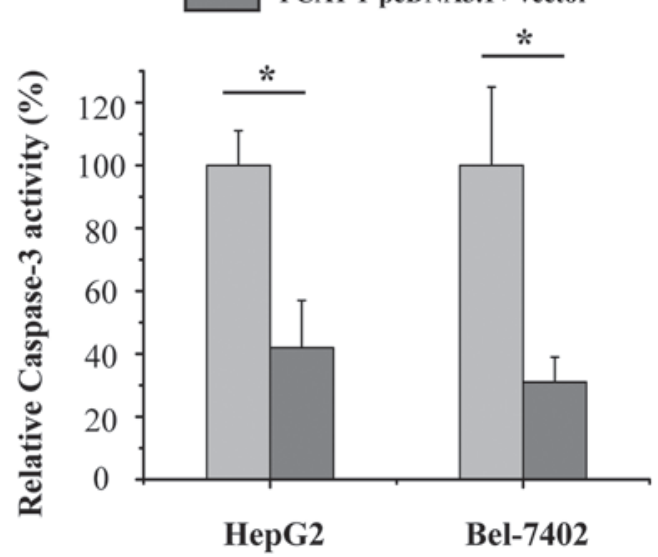

Figure 5. Effect of PCAT-1 on apoptosis in HCC cells. After 48 h transfection of PCAT-1 shRNA or negative control shRNA, and PCAT-1-pcDNA3.1 + vector or pcDNA3.1 + empty vector, the changes in cell apoptosis were determined by ELISA. (A) The relative activity of caspase-3 was increased in HepG2 and Bel-7402 cells treated with PCAT-1 shRNA. (B) The relative activity of caspase-3 was decreased in HepG2 and Bel-7402 cells treated with PCAT-1-pcDNA3.1 + vector. Data were expressed as the mean \pm standard deviation $(n=3)$. All experiments were performed three times. ${ }^{*}<0.05$. PCAT1, prostate cancer-asssociated transcript 1; HCC, hepatocellular carcinoma; shRNA, small hairpin RNA. 
PCAT-1-pcDNA3.1 + vector-transfected HCC cells. These results suggest that PCAT-1 has an oncogenic role in the progression and development of HCC.

In conclusion, PCAT-1 exerts an oncogenic effect in HCC. Further investigation is required to clarify the underlying molecular mechanisms of PCAT-1 as a candidate biomarker for HCC therapeutic strategies.

\section{References}

1. Jemal A, Bray F, Center MM, Ferlay J, Ward E and Forman D: Global cancer statistics. CA Cancer J Clin 61: 69-90, 2011.

2. El-Serag HB and Rudolph KL: Hepatocellular carcinoma: Epidemiology and molecular carcinogenesis. Gastroenterology 132: 2557-2576, 2007.

3. Tang ZY, Ye SL, Liu YK, Qin LX, Sun HC, Ye QH, Wang L, Zhou J, Qiu SJ, Li Y, et al: A decade's studies on metastasis of hepatocellular carcinoma. J Cancer Res Clin Oncol 130: 187-196, 2004.

4. Yang $Y$, Nagano $H$, Ota $H$, Morimoto $O$, Nakamura $M$, Wada $H$, Noda T, Damdinsuren B, Marubashi S, Miyamoto A, et al: Patterns and clinicopathologic features of extrahepatic recurrence of hepatocellular carcinoma after curative resection. Surgery 141: 196-202, 2007.

5. Luk JM, Burchard J, Zhang C, Liu AM, Wong KF, Shek FH, Lee NP, Fan ST, Poon RT, Ivanovska I, et al: DLK1-DIO3 genomic imprinted microRNA cluster at $14 \mathrm{q} 32.2$ defines a stemlike subtype of hepatocellular carcinoma associated with poor survival. J Biol Chem 286: 30706-30713, 2011.

6. Clark T, Maximin S, Meier J, Pokharel S and Bhargava P: Hepatocellular Carcinoma: Review of epidemiology, screening, imaging diagnosis, response assessment and treatment. Curr Probl Diagn Radiol 44: 479-486, 2015.

7. Huang B and Zhang R: Regulatory non-coding RNAs: Revolutionizing the RNA world. Mol Biol Rep 41: 3915-3923, 2014.

8. Du Y, Wang L, Wu H,Zhang Y, Wang K and Wu D: MicroRNA-141 inhibits migration of gastric cancer by targeting zinc finger E-box-binding homeobox 2. Mol Med Rep 12: 3416-3422, 2015.

9. Wang T, Liu Y, Yuan W, Zhang L, Zhang Y, Wang Z, Zhou X, Zhou H, Chu T, Hao Y, et al: Identification of microRNAome in rat bladder reveals miR-1949 as a potential inducer of bladder cancer following spinal cord injury. Mol Med Rep 12: 2849-2857, 2015.

10. Li L and Ma HQ: MicroRNA216a inhibits the growth and metastasis of oral squamous cell carcinoma by targeting eukaryotic translation initiation factor 4B. Mol Med Rep 12: 3156-3162, 2015.

11. Mercer TR, Dinger ME and Mattick JS: Long non-coding RNAs: Insights into functions. Nat Rev Genet 10: 155-159, 2009.

12. Lee JT and Bartolomei MS: X-inactivation, imprinting and long noncoding RNAs in health and disease. Cell 152: 1308-1323, 2013

13. Gibb EA, Brown CJ and Lam WL: The functional role of long non-coding RNA in human carcinomas. Mol Cancer 10: 38, 2011.
14. Liu J, Shen L, Yao J, Li Y, Wang Y, Chen H and Geng P: Forkhead box $\mathrm{C} 1$ promoter upstream transcript, a novel long non-coding RNA, regulates proliferation and migration in basal-like breast cancer. Mol Med Rep 11: 3155-3159, 2015

15. Wang Y, Yao J, Meng H, Yu Z, Wang Z, Yuan X, Chen H and Wang A: A novel long non-coding RNA, hypoxia-inducible factor- $2 \alpha$ promoter upstream transcript, functions as an inhibitor of osteosarcoma stem cells in vitro. Mol Med Rep 11: 2534-2540, 2015.

16. Wang Y, Xu G, Chen W, Pan Q, Huang K, Pan J, Zhang W and Chen J: Detection of long-chain non-encoding RNA differential expression in non-small cell lung cancer by microarray analysis and preliminary verification. Mol Med Rep 11: 1925-1932, 2015.

17. Shi X, Sun M, Liu H, Yao Y and Song Y: Long non-coding RNAs: A new frontier in the study of human diseases. Cancer Lett 339: 159-166, 2013

18. Prensner JR, Iyer MK, Balbin OA, Dhanasekaran SM, Cao Q, Brenner JC, Laxman B, Asangani IA, Grasso CS, Kominsky HD, et al: Transcriptome sequencing across a prostate cancer cohort identifies PCAT-1, an unannotated lincRNA implicated in disease progression. Nat Biotechnol 29: 742-749, 2011.

19. Ge X, Chen Y, Liao X, Liu D, Li F, Ruan H and Jia W: Overexpression of long noncoding RNA PCAT-1 is a novel biomarker of poor prognosis in patients with colorectal cancer. Med Oncol 30: 588, 2013.

20. Shi WH, Wu QQ, Li SQ, Yang TX, Liu ZH, Tong YS, Tuo L, Wang S and Cao XF: Upregulation of the long noncoding RNA PCAT-1 correlates with advanced clinical stage and poor prognosis in esophageal squamous carcinoma. Tumour Biol 36: 2501-2507, 2015.

21. Schmittgen TD and Livak KJ: Analyzing real-time PCR data by the comparative C(T) method. Nat Protoc 3: 1101-1108, 2008.

22. Yang X, Xie X, Xiao YF, Xie R, Hu CJ, Tang B, Li BS and Yang SM: The emergence of long non-coding RNAs in the tumorigenesis of hepatocellular carcinoma. Cancer Lett 360: 119-124, 2015.

23. Sun J, Bie B, Zhang S, Yang J and Li Z: Long non-coding RNAs: Critical players in hepatocellular carcinoma. Int J Mol Sci 15: 20434-20448, 2014

24. Huang JL, Zheng L, Hu YW and Wang Q: Characteristics of long non-coding RNA and its relation to hepatocellular carcinoma. Carcinogenesis 35: 507-514, 2014.

25. Prensner JR, Chen W, Iyer MK, Cao Q, Ma T, Han S, Sahu A, Malik R, Wilder-Romans K, Navone N, et al: PCAT-1, a long noncoding RNA, regulates BRCA2 and controls homologous recombination in cancer. Cancer Res 74: 1651-1660, 2014.

26. Prensner JR, Chen W, Han S, Iyer MK, Cao Q, Kothari V, Evans JR, Knudsen KE, Paulsen MT, Ljungman M, et al: The long non-coding RNA PCAT-1 promotes prostate cancer cell proliferation through cMyc. Neoplasia 16: 900-908, 2014.

27. Liu L, Liu Y, Zhuang C, Xu W, Fu X, Lv Z, Wu H, Mou L, Zhao G, Cai Z and Huang W: Inducing cell growth arrest and apoptosis by silencing long non-coding RNA PCAT-1 in human bladder cancer. Tumour Biol 36: 7685-7689, 2015. 